\title{
Effective Fingerprint Classification by Localized Models of Support Vector Machines
}

\author{
Jun-Ki Min, Jin-Hyuk Hong, and Sung-Bae Cho \\ Department of Computer Science, Yonsei University, \\ Biometrics Engineering Research Center, \\ 134 Shinchon-dong, Sudaemoon-ku, Seoul 120-749, Korea \\ \{loomlike, hjinh\}@sclab.yonsei.ac.kr, sbcho@cs.yonsei.ac.kr
}

\begin{abstract}
Fingerprint classification is useful as a preliminary step of the matching process and is performed in order to reduce searching time. Various classifiers like support vector machines (SVMs) have been used to fingerprint classification. Since the SVM which achieves high accuracy in pattern classification is a binary classifier, we propose a classifier-fusion method, multiple decision templates (MuDTs). The proposed method extracts several clusters of different characteristics from each class of fingerprints and constructs localized classification models in order to overcome restrictions to ambiguous fingerprints. Experimental results show the feasibility and validity of the proposed method.
\end{abstract}

\section{Introduction}

Fingerprint classification is a technique that classifies fingerprints into the predefined categories according to the characteristics of the image. It is useful for an automated fingerprint identification system (AFIS) as a preliminary step of the matching process and is performed in order to reduce searching time. Fig. 1 shows the examples of fingerprint classes.

Various classifiers, such as neural networks, k-nearest neighbors, and SVMs, have been widely used in fingerprint classification [1]. Since the SVM which shows good performance in pattern classification was originally designed for binary classification, it requires a combination method in order to classify multiclass fingerprints [2].

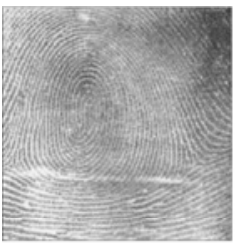

(a)

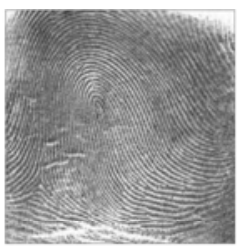

(b)

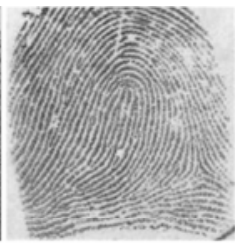

(c)

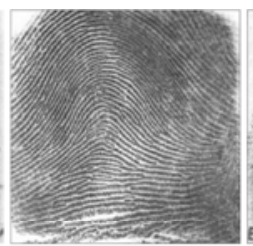

(d)

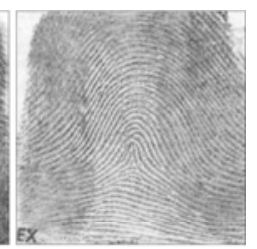

(e)

Fig. 1. Five fingerprint classes in the NIST database 4. (a) Whorl, (b) Right loop, (c) Left loop, (d) Arch, (e) Tented arch. 
Many classifier-fusion methods have been investigated for the purpose of extending binary classification to multiclass classification or for improving classification accuracy [4]. Especially, the decision templates (single-DTs) have produced good performance in recent applications [5]. Since this method abstracts the outputs of the classifiers to a template, there is a limitation of applying it to complex problems with ambiguous samples such as fingerprints [6]. For the effective combination of SVMs in order to classify fingerprints, we propose multiple decision templates (MuDTs) that localized fusion models with clustering algorithm. The MuDTs decompose one class into several clusters to produce decision templates of each cluster. The proposed method is validated on the NIST database 4 using FingerCode features.

\section{Related Works}

\subsection{The FingerCode}

The FingerCode, as proposed by Jain in 1999, was extracted from NIST database 4 using a filter-based method. The algorithm set a registration point in a given fingerprint image and tessellated it into 48 sectors. Then, it transformed the image using the Gabor filter of four directions $\left(0^{\circ}, 45^{\circ}, 90^{\circ}\right.$, and $\left.135^{\circ}\right)$. Ridges parallel to each filter direction were accentuated, and ridges not parallel to the directions were blurred (Fig. 2). Standard deviations were computed on 48 sectors for each of the four transferred images in order to generate the 192-dimensional feature vector called FingerCode. Jain achieved $90 \%$ accuracy at a $1.8 \%$ rejection rate with two stage classification of K-NN/neural networks using these features [3].

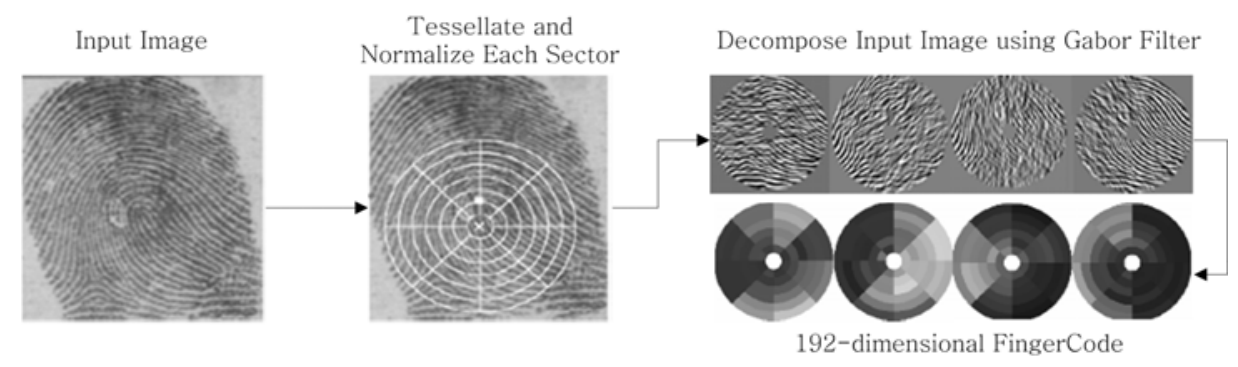

Fig. 2. Flow diagram of the FingerCode feature vector [3]

\subsection{Support Vector Machines}

The SVM is a technique for binary classification in the field of pattern recognition. This technique maps an input sample to a high-dimensional feature space and finds the optimal hyperplane that minimizes the recognition error for the training data using the non-linear transformation function.

Let $n$ be the number of training samples. For the $i$ th sample $x_{i}$ with class label $c_{i} \in\{1,-1\}$, the SVM calculates 


$$
f(x)=\sum_{i=1}^{n} \alpha_{i} c_{i} K\left(x, x_{i}\right)+b, \quad K\left(x, x_{i}\right)=\Phi(x) \cdot \Phi\left(x_{i}\right) .
$$

Coefficient $\alpha_{i}$ in Eq. (1) is non-zero when $x_{i}$ is a support vector that composes the hyperplane. Under all other conditions, it is zero. The kernel function $K\left(x, x_{i}\right)$ is easily computed by defining an inner product of the non-linear mapping function.

To classify fingerprints using SVMs, decomposition strategies such as one-vs-all, pairwise, and complete-code are needed [7].

\subsection{The Decision Templates}

The decision templates (single-DTs) generate templates of each class by averaging the decision profiles (DPs) for the training samples. For the $M$-class problem with $L$ classifiers, $D P\left(x_{i}\right)$ of the $i$ th sample is

$$
D P\left(x_{i}\right)=\left[\begin{array}{ccc}
d_{1,1}\left(x_{i}\right) & \ldots & d_{1, M}\left(x_{i}\right) \\
\vdots & d_{y, z}\left(x_{i}\right) & \vdots \\
d_{L, 1}\left(x_{i}\right) & \ldots & d_{L, M}\left(x_{i}\right)
\end{array}\right],
$$

where $d_{y, z}\left(x_{i}\right)$ is the degree of support given by the $y$ th classifier for the sample $x_{i}$ of the class $z$. When DPs are generated from the training data, Eq. (3) estimates the decision template $D T_{c}$ of the class $c$.

$$
D T_{c}=\left[\begin{array}{ccc}
d t_{c}(1,1) & \ldots & d t_{c}(1, M) \\
\vdots & d t_{c}(y, z) & \vdots \\
d t_{c}(L, 1) & \ldots & d t_{c}(L, M)
\end{array}\right], \quad d t_{c}(y, z)=\frac{\sum_{i=1}^{n} i n d_{c}\left(x_{i}\right) d_{y, z}\left(x_{i}\right)}{\sum_{i=1}^{n} i n d_{c}\left(x_{i}\right)}
$$

$\operatorname{Ind}_{c}\left(x_{i}\right)$ has a value of 1 if $x_{i}{ }^{\prime}$ s class is $c$, otherwise it has a value of zero.

In the test stage, it computes the distance between the DP of a new sample and the decision templates of each class. The class label is decided as the class of the most similar decision templates [5].

\section{Multiple Decision Templates}

In order to construct the MuDTs, we composed decision profiles with 5 one-vs-all SVMs (whorl, right loop, left loop, arch, and tented arch versus all). Decision profiles of each class $D P_{\text {whorl }}(x), \ldots, D P_{\text {tented arch }}(x)$ were clustered with a SOM algorithm (Eq. (4)). Each $D P(x)$ mapped a sample to the cluster $(k, l)$ using Euclidean distance, with $w_{i, j}$ as the weight of the $(i, j)$ th cluster [8].

$$
\left\|D P(x)-w_{k, l}\right\|=\min _{i, j=1, \ldots, N}\left\{\left\|D P(x)-w_{i, j}\right\|\right\}
$$




\begin{tabular}{|c|}
\hline Whorl-vs-all \\
\hline Right loop-vs-all \\
\hline Left loop-vs-all \\
\hline Arch-vs-all \\
\hline Tented arch-vs-all \\
\hline
\end{tabular}

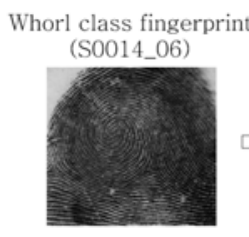

Decision profile of one-vs-all SVMs
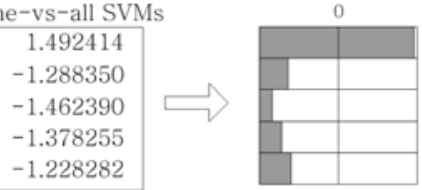

Fig. 3. A template of one-vs-all SVMs with its graphical representation

A decision template $D T_{c}^{k, l}$, which is the template of a cluster $(k, l)$ of class $c$, was computed by Eq. (5). $\operatorname{Ind} d_{c}^{k, l}\left(x_{i}\right)$ refers to an indicator function of 1 if $x_{i}$ belongs to the $(k, l)$ th cluster of class $c$. If this is not the case, it refers to zero.

$$
D T_{c}^{k, l}=\left[\begin{array}{ccc}
d t_{c}^{k, l}(1,1) & \ldots & d t_{c}^{k, l}(1, M) \\
\vdots & d t_{c}^{k, l}(y, z) & \vdots \\
d t_{c}^{k, l}(L, 1) & \cdots & d t_{c}^{k, l}(L, M)
\end{array}\right], \quad d t_{c}^{k, l}(y, z)=\frac{\sum_{i=1}^{n} i n d_{c}^{k, l}\left(x_{i}\right) d_{y, z}\left(x_{i}\right)}{\sum_{i=1}^{n} i n d_{c}^{k, l}\left(x_{i}\right)}
$$

Since the SVM is a binary classifier, we represented the output of a classifier to one column with positive and negative signs (Fig. 3). Sixteen decision templates of a class were estimated by clustering $4 \times 4$ SOM as shown in Fig. 4.

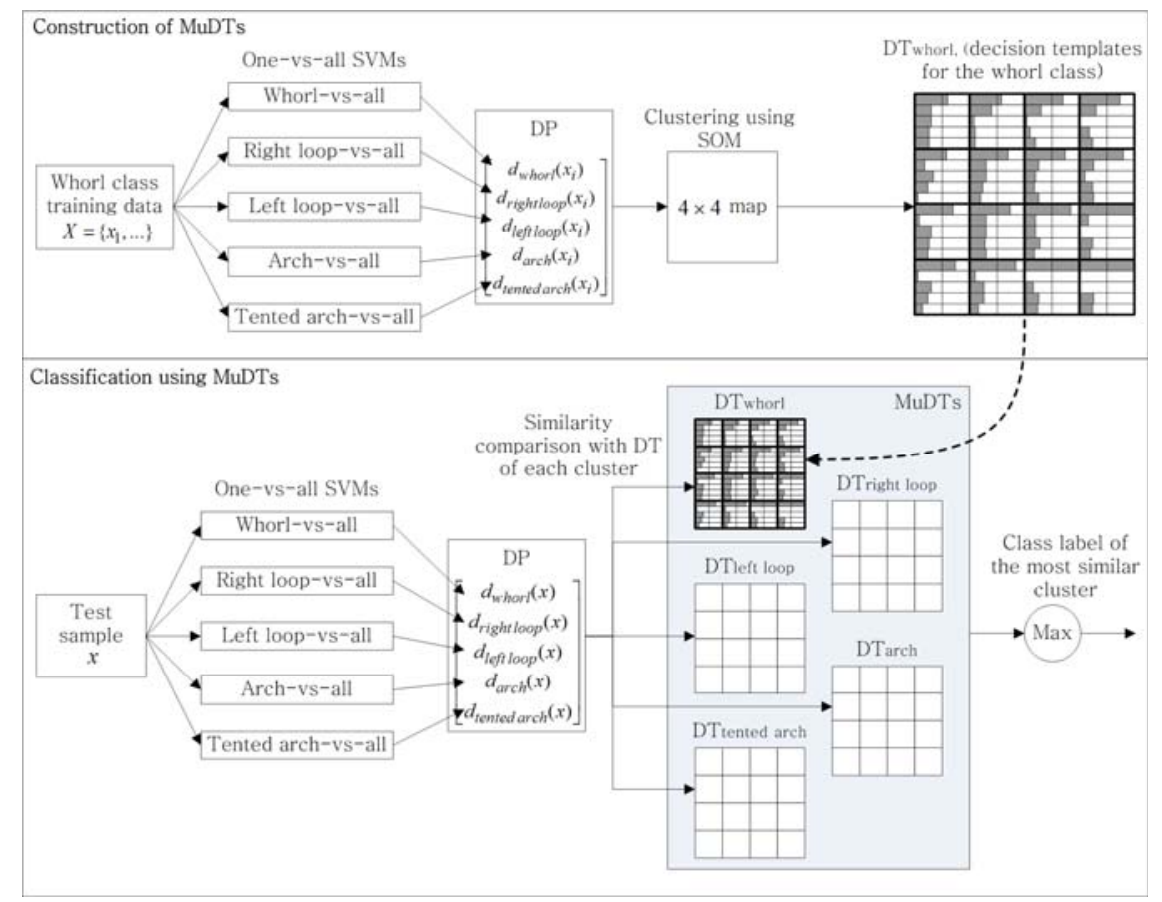

Fig. 4. Construction and classification of $4 \times 4$ MuDTs (case of whorl class) 
The classification process of the MuDTs is similar to that used with single-DTs'. The distance between the decision profile of a new sample and each decision template of clusters is calculated (Fig. 4), and then the sample is classified into the class that contains the most similar clusters. In this paper, the Euclidean distance (Eq. (6)) is used to measure the similarity for its simplicity and good performance [5].

$$
d s t_{c}^{i, j}(x)=\sqrt{\sum_{y=1}^{L} \sum_{z=1}^{M}\left(d t_{c}^{i, j}(y, z)-d_{y, z}(x)\right)^{2}}, \min _{c=1, \ldots, M}\left(\min _{i, j=1, \ldots, n} d s t_{c}^{i, j}(x)\right)
$$

\section{Experimental Results}

\subsection{Experimental Environments}

We have verified the proposed method on the NIST database 4. The first set of impressions of the fingerprints (F0001 F2000) were used as the training set while the second set of impressions of the fingerprints (S0001 S2000) were used as the test set. Jain's FingerCode features were used after normalization $(+1 \sim-1)$. The FingerCode rejected a few fingerprint images in both the training set (1.4\%) and the test set (1.8\%) [3]. The LIBSVM package (available at http://www.csie.ntu.edu.tw/ cjlin/libsvm) was used for the SVM classifiers. The Gaussian kernel with $\sigma^{2}=0.0625$ was selected based on the experiment.

\subsection{MuDTs Versus DTs}

The MuDTs of the one-vs-all (OVA) SVMs yielded an accuracy of 90.4\% for the 5class classification task. For the 4-class classification task, 94.9\% was achieved. The confusion matrices of the one-vs-all SVMs combined with the single-DTs and MuDTs with the Euclidean distance are shown in Table 1 and Table 2. Because the MuDTs produce multiple classification models for one class, they classify ambiguous fingerprint images more accurately than single-DTs (Fig. 5).

Table 1. Confusion matrix for the single-DTs of OVA SVMs

\begin{tabular}{llllll}
\hline & $\mathrm{W}$ & $\mathrm{R}$ & $\mathrm{L}$ & $\mathrm{A}$ & $\mathrm{T}$ \\
\hline $\mathrm{W}$ & 380 & 6 & 8 & 0 & 0 \\
$\mathrm{R}$ & 7 & 357 & 1 & 6 & 21 \\
$\mathrm{~L}$ & 7 & 0 & 363 & 13 & 13 \\
$\mathrm{~A}$ & 1 & 2 & 1 & 347 & 60 \\
$\mathrm{~T}$ & 1 & 8 & 9 & 37 & 316 \\
\hline
\end{tabular}

Table 2. Confusion matrix for the MuDTs of OVA SVMs

\begin{tabular}{llllll}
\hline & $\mathrm{W}$ & $\mathrm{R}$ & $\mathrm{L}$ & $\mathrm{A}$ & $\mathrm{T}$ \\
\hline $\mathrm{W}$ & 380 & 6 & 7 & 0 & 1 \\
$\mathrm{R}$ & 9 & 369 & 1 & 5 & 17 \\
$\mathrm{~L}$ & 8 & 0 & 366 & 14 & 10 \\
$\mathrm{~A}$ & 1 & 4 & 1 & 356 & 50 \\
$\mathrm{~T}$ & 1 & 10 & 6 & 38 & 304 \\
\hline
\end{tabular}

\subsection{Comparison with Other Methods}

The winner-takes-all, ECCs, BKS, and single-DTs methods were compared with the MuDTs. The Euclidean distance was used for ECCs, single-DTs, and MuDTs. For the 


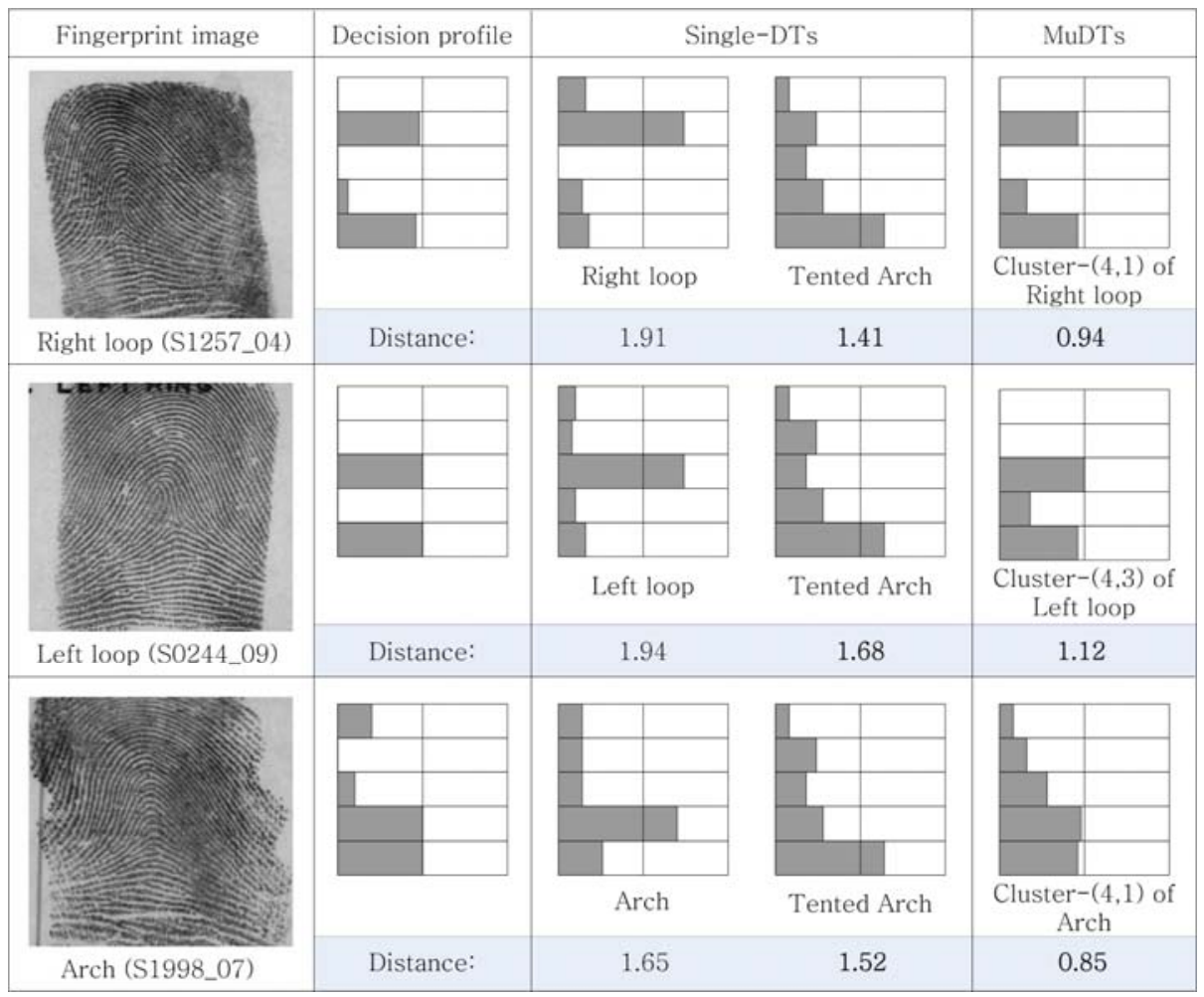

Fig. 5. Classification of ambiguous fingerprints

BKS method, when ties or new output patterns occurred, the winner-takes-all method was alternatively used. As shown in Table 3, the MuDTs achieved the highest accuracy of $89.5 \% \sim 90.4 \%$. Given the simplicity of the SOM algorithm with the low-dimension vector, despite the additional step for clustering at the training phase, there is nearly no difference between the classification times of the MuDTs and single-DTs. It took about $60 \mathrm{~ms}$ on a Pentium $4(2.4 \mathrm{GHz})$ machine to train the SOM with 2,000 fingerprints which can be ignored, compared to the training time of the SVMs.

Table 3. The accuracies of various classifier fusion schemes (\%)

\begin{tabular}{llll}
\hline Fusion methods & One-vs-all & Pairwise & Complete-code \\
\hline Winner-takes-all & 90.1 & 87.7 & 90.0 \\
ECCs & 90.1 & 88.6 & 90.0 \\
BKS & 88.8 & 89.4 & 89.3 \\
Single-DTs & 89.8 & 88.3 & 89.5 \\
MuDTs & 90.4 & 89.5 & 90.3 \\
\hline
\end{tabular}




\section{Conclusion}

This paper has proposed an effective classifier fusion method (MuDTs) to classify ambiguous fingerprint images which show more than one characteristic in terms of fingerprint class. The outputs of one-vs-all SVMs for the training data were clustered by the SOM to decompose the class into several clusters to separate and examine diverse characteristics. The localized decision templates were estimated for each cluster, and then the MuDTs were constructed. Experiments were performed on the NIST database 4 using FingerCodes. We achieved $90.4 \%$ for 5-class classification with $1.8 \%$ rejection, and $94.9 \%$ for 4 -class classification. Experimental results show the effectiveness of the multiple templates method with higher accuracy than other methods. In future work, we will investigate effective classifier decomposition methods with appropriate cluster maps to maximize the effectiveness of the MuDTs.

Acknowledgements. This work was supported by the Korea Science and Engineering Foundation (KOSEF) through the Biometrics Engineering Research Center (BERC) at Yonsei University. We would like to thank Prof. Anil Jain and Dr. Salil Prabhakar for providing the FingerCode data.

\section{References}

1. A. Senior, "A combination fingerprint classifier," IEEE Trans. Pattern Analysis and Machine Intelligence, vol. 23, no. 10, pp. 1165-1174, 2001.

2. Y. Yao, et al., "Combining flat and structured representations for fingerprint classification with recursive neural networks and support vector machines," Pattern Recognition, vol. 36, no. 2, pp. 397-406, 2003.

3. A. K. Jain, et al., "A multichannel approach to fingerprint classification," IEEE Trans. Pattern Analysis and Machine Intelligence, vol. 21, no. 4, pp. 348-359, 1999.

4. L. I. Kuncheva, Combining Pattern Classifiers, Wiley-Interscience, 2004.

5. L. I. Kuncheva, et al., "Decision templates for multiple classifier fusion: An experimental comparison," Pattern Recognition, vol. 34, no. 2, pp. 299-314, 2001.

6. R. Cappelli, et al., "Fingerprint classification by directional image partitioning," IEEE Trans. Pattern Analysis and Machine Intelligence, vol. 21, no. 5, pp. 402-421, 1999.

7. R. M. Rifkin and A. Klautau, "In defence of one-vs-all classification," Jnl. of Machine Learning Research, vol. 5, pp. 101-141, 2004.

8. K. Obermayer and T. J. Sejnowski, Self-Organizing Map Formation Foundations of Neural Computation, The MIT Press, 2001. 